\title{
Advantages of Cs-correctors for Spectrometry in STEM
}

M. Watanabe*, D.W. Ackland*, A. Burrows*, C.J. Kiely*, D.B. Williams*, M. Kanno** and R. Hynes**

*Department of Materials Science and Engineering and Center for Advanced Materials and Nanotechnology, Lehigh University, Bethlehem. PA, USA.

**JEOL USA Ltd., 11 Dearborn Rd., Peabody, MA, USA.

Recent theoretical calculations and practical experiments have proven that high-angle annular dark-field (HA-ADF) imaging is significantly improved due to the incident probe refined by a spherical aberration corrector $\left(\mathrm{C}_{\mathrm{s}}\right.$-corrector) in scanning transmission electron microscopes (STEMs) [1]. The Oak Ridge group has achieved the sub- $\AA$ image resolution using a $300 \mathrm{keV}$ Cs-corrected STEM [2]. For microanalysis via electron energy-loss spectrometry (EELS) and/or X-ray energy dispersive spectrometry (XEDS), the major benefit due to the $\mathrm{C}_{\mathrm{s}}$ corrector is also improvement of spatial resolution in analysis. For the EELS analysis, it is possible to achieve atomic-level spatial resolution even in conventional STEM instruments and further improvements have been demonstrated by the Cs-corrected STEM [3]. Figure 1 shows a HA-ADF STEM image of $\mathrm{Si}<110>$ (a) and an EELS spectrum around $\mathrm{Si} \mathrm{K}$ edge (at $1839 \mathrm{eV}$ ) (b) obtained with the beam current of $50 \mathrm{pA}$ in a $200 \mathrm{keV}$ JEM-2200FS STEM/TEM at Lehigh University, which is equipped with a CEOS STEM $\mathrm{C}_{\mathrm{s}}$-corrector. It is possible to measure higher core-loss spectrum within a reasonable acquisition time (10 $\mathrm{s}$ in this case) while maintaining the atomic resolution $(1.36 \AA)$.

In the STEM-XEDS approach, much higher beam currents are required to generate sufficient $\mathrm{X}$-ray signals. Because of enlargement of the probe due to the higher beam current, the atomic-column spatial resolution is not achievable in conventional STEMs. Recently, a VG HB $603(300 \mathrm{keV})$ dedicated STEM at Lehigh is upgraded by installing a Nion $\mathrm{C}_{\mathrm{s}}$ corrector. Figure 2 shows simulated intensity distributions of the incident probes at $1 \mathrm{nA}$ in conventional and Cs-corrected configurations of the HB 603. By the Cs-corrector, the incident probe can be reduced by a factor of 2.5 (from $1.5 \mathrm{~nm}$ to $0.6 \mathrm{~nm}$ ) even with the same beam current. The theoretical calculation for XEDS shows that the sub-nm spatial resolution is achievable with the $1 \mathrm{nA}$ probe (Fig. 3(a)). Conversely, the beam current can be increased considerably in the same beam size by the Cs-corrector, and hence the analytical sensitivity can also be improved. Fig. 3(b) shows an expected improvement in analytical sensitivity by using Cs-corrected STEMs. It is considered that all these recent developments related to analytical STEMs may permit atomic-level analytical resolution in X-ray mapping. 
References

[1] O. Krivanek et al., Ultramicroscopy 78(1999) 1.

[2] P.D. Nellist et al., Science, 305 (2004) 1741.

[3] K. van Benthem \& S.J. Pennycook, Microsc. Microanal. 10 (Suppl 2) (2004) 206.

[4] This research was supported by NSF DMR-9626279, DMR-0320906, DMR-0304738 and Bechtel Bettis Laboratories.

Fig. 1
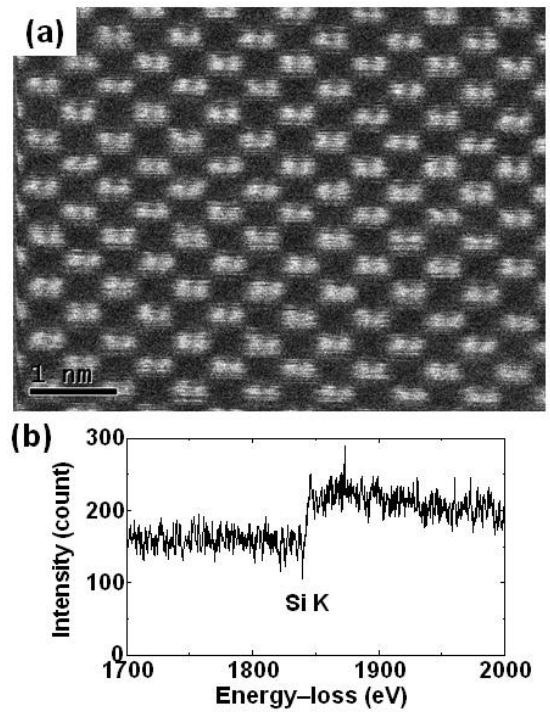
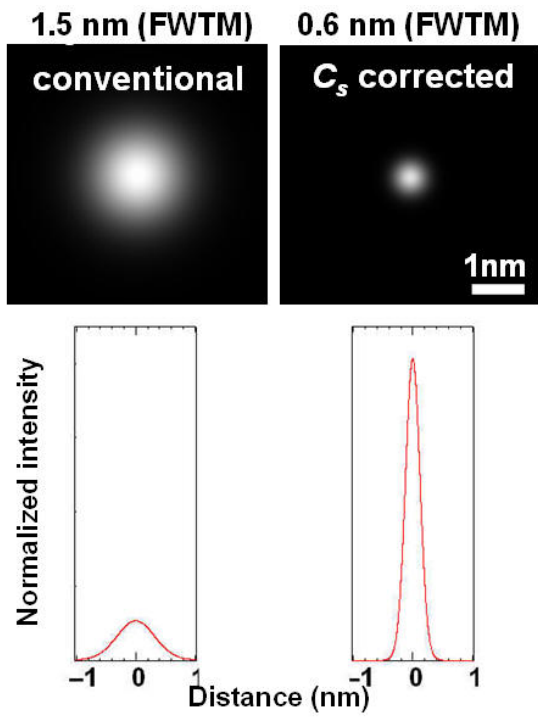

Fig. 2

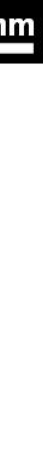

Fig. 1. a HA-ADF STEM image of $\mathrm{Si}<110>$ (a) and an EELS spectrum around the Si K edge measured in the JEM-2200FS Cs-corrected STEM/TEM at Lehigh University.

Fig. 2. Simulated images and profiles of the incident probes in conventional (left) and Cs-corrected (right) configurations of the VG HB 603 dedicated STEM.

(a)

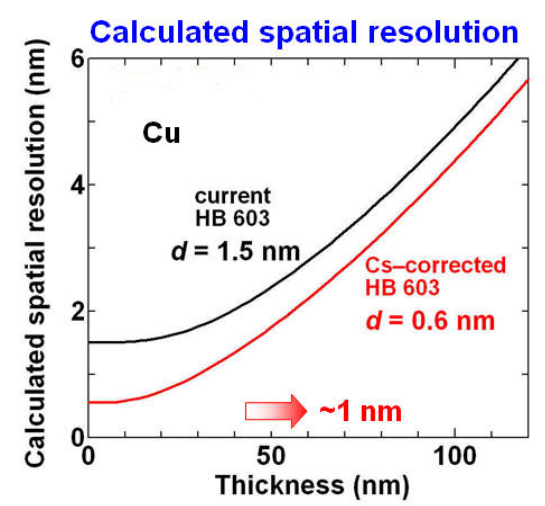

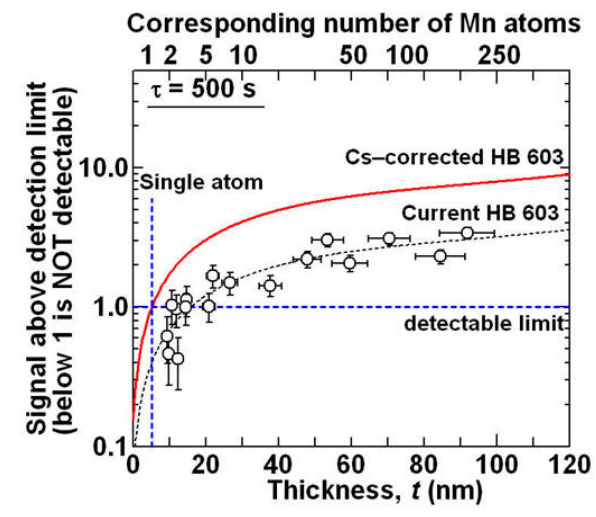

(b)

Fig. 3 (a) Simulated spatial resolution of XEDS analysis of a $\mathrm{Cu}-5 \mathrm{wt} \% \mathrm{Mn}$ thin specimen in the conventional (dashed line) and the $\mathrm{C}_{\mathrm{s}}$-corrected HB 603 (solid line). (b) X-ray signal quality as a function of thickness for $\mathrm{Cu}-0.12 \% \mathrm{Mn}$, measured in the conventional HB 603 (circles) and calculated for the $\mathrm{C}_{\mathrm{s}}$-corrected HB 603. 